\title{
Patterns of cervical lymph node metastasis in supraglottic laryngeal cancer and therapeutic implications of surgical staging of the neck
}

\author{
Cornelius H. L. Kürten ${ }^{1} \cdot$ Eleni Zioga ${ }^{1} \cdot$ Thomas Gauler $^{2} \cdot$ Martin Stuschke $^{2} \cdot$ Maja Guberina $^{2}$ - Johannes M. Ludwig ${ }^{3}$. \\ Eric Deuss ${ }^{1} \cdot$ Stefan Mattheis ${ }^{1} \cdot$ Stephan Lang ${ }^{1} \cdot$ Timon Hussain $^{1}$ (I)
}

Received: 7 February 2021 / Accepted: 11 March 2021 / Published online: 27 March 2021

(c) The Author(s) 2021

\begin{abstract}
Purpose Accurate therapeutic management of the neck is a challenge in patients with supraglottic laryngeal cancer. Nodal metastasis is common at all disease stages, and treatment planning relies on clinical staging of the neck, for both surgical and non-surgical treatment. Here, we compared clinical and surgical staging results in supraglottic carcinoma patients treated with primary surgery to assess the accuracy of pre-therapeutic clinical staging and guide future treatment decisions.

Methods Retrospective analysis of clinical, pathological, and oncologic outcome data of 70 patients treated with primary surgery and bilateral neck dissection for supraglottic laryngeal cancer. Patients where clinical and pathological neck staging results differed, were identified and analyzed in detail.

Results On pathologic assessment, patients with early stage (pT1/2) primaries showed cervical lymph node metastases in $55 \%(n=17 / 31)$ of cases, compared to $67 \%(n=26 / 39)$ of patients with pT3/4 tumors. In $24 \%(n=17 / 70)$ of all patients, $\mathrm{cN}$ status differed from $\mathrm{pN}$ status, resulting in an upstaging in $16 \%$ of cases $(n=11 / 70)$ and a downstaging in $9 \%(n=6 / 70)$ of cases. $14 \%$ of patients with cN0 status had occult metastases $(n=5 / 30)$. As assessed by a retrospective tumor board, in case of a non-surgical treatment approach, the inaccurate clinical staging of the neck would have led to an over- or undertreatment of the neck in $20 \%(n=14 / 70)$ of all patients.

Conclusion Our data re-emphasize the high cervical metastasis rates of supraglottic laryngeal cancer across all stages. Inaccurate clinical staging of the neck is common and should be taken into consideration when planning treatment.
\end{abstract}

Keywords Head and neck cancer $\cdot$ Laryngeal cancer $\cdot$ Neck dissection $\cdot$ Transoral robotic surgery $\cdot$ Staging

\section{Introduction}

Due to the extensive regional lymphatic network, supraglottic laryngeal carcinomas have a tendency to develop neck metastases at all disease stages, hereby negatively impacting patients' prognosis and influencing treatment decisions [1, 2]. For patients treated with a primary surgical approach, nodal metastasis is routinely managed by neck dissection,

Timon Hussain

timon.hussain@uk-essen.de

1 Department of Otorhinolaryngology, Head and Neck Surgery, University Hospital Essen, University of DuisburgEssen, Hufelandstraße 55, 45147 Essen, Germany

2 Department of Radiation Oncology, University Hospital Essen, University of Duisburg-Essen, Essen, Germany

3 Department of Radiology, University Hospital Essen, University of Duisburg-Essen, Essen, Germany oftentimes followed by adjuvant radiotherapy. While ipsilateral elective neck dissection is generally recommended as part of a primary surgical approach for supraglottic carcinomas by current guidelines, the question whether bilateral neck dissection of the clinically N0 neck is warranted has been debated controversially [3-5]. For head and neck cancer in general, based on decision analysis models [6], a $20 \%$ chance of occult metastasis has been proposed to be a justifiable threshold for elective neck dissection. Given the significant changes in neck dissection technique in the past decades [7], today, even occult metastasis rates lower than $20 \%$ have been deemed appropriate to justify elective neck dissection of the respective levels at risk $[5,8]$. This changed risk-benefit analysis is mainly driven by a more selective and functionally orientated neck dissection classification and surgical technique as well as improved imaging techniques [8-10]. 
At our institution, in anticipation of high rates of occult nodal metastasis, all patients undergoing primary surgery for supraglottic laryngeal carcinomas also routinely underwent bilateral neck dissection. Based hereon, we compared the pre-operative clinical neck staging $(\mathrm{cN})$ results, as assessed by ultrasound, and CT scans, to post-operative neck staging results, as assessed by pathology (surgical staging, $\mathrm{pN}$ ), hereby determining the accuracy of clinical staging of the neck and the rate of occult metastasis in our patient collective. Patients undergoing a primary surgical approach including bilateral neck dissection have the benefit of a definitive pathological nodal status after surgery which guides adjuvant treatment planning, [11] i.e., the addition of radiotherapy if nodal involvement is shown [12], and radiochemotherapy with the inclusion of cisplatin if extracapsular spread is established, or the resection is incomplete [13]. On the other hand, in patients undergoing a primary non-surgical treatment approach, clinical nodal staging determines the entire therapeutic regimen and is, therefore, of particular interest. Potential discrepancy rates between clinical and pathological staging results must be kept in mind during therapy planning to avoid over/undertreatment.

\section{Materials and methods}

\section{Patients}

We selected patients who underwent primary surgery for supraglottic laryngeal cancer at our institution between 2004 and 2019. Inclusion criteria were complete staging and initial treatment at our cancer center, no secondary malignancy at the time of diagnosis, availability of clinical as well as pathological staging data and the treatment recommendation of our interdisciplinary tumor board to perform primary tumor resection and bilateral neck dissection with curative intent. Routine staging included contrast-enhanced CT scans of the head and neck, contrast enhanced CT scan of the thorax to rule out lung metastasis or second primaries, ultrasound of the abdomen to rule out abdominal metastasis or second primaries and ultrasound of the neck. All clinical and pathological parameters were recorded by chart review. Staging was determined according to the current staging system at time of diagnosis (AJCC Staging Manual 6th Edition up to 2009, AJCC Staging Manual 7th Edition up to 2017, AJCC Staging Manual 8th Edition starting up to 2019).

\section{Retrospective comparison of clinical and pathological neck staging}

An experienced radiologist and an experienced radiation oncologist blinded to $\mathrm{pN}$-status re-assessed the clinical node status of patients with divergent $\mathrm{cN}$ vs. pN. In $3 / 17$ cases, the head and neck CT scan was no longer available in the electronic radiology database and we therefore, relied on the written report at the time of diagnosis. We conducted a virtual retrospective tumor board to determine the impact of the postoperative $\mathrm{pN}$-status on hypothetical treatment decision had the patient received primary radiotherapy.

\section{Statistical analysis and data visualization}

Survival analysis was performed using GraphPad Prism 8 for Windows (Version 8.4.3, GraphPad Software, San Diego, CA, USA). 67 patients, for which survival data were available, were included. The sankey diagram was created using SankeyMATIC (http://sankeymatic.com). Figure 2, for easier visualization, shows the stages $\mathrm{c} / \mathrm{pN} 3 \mathrm{a}$ and $\mathrm{c} / \mathrm{p} 3 \mathrm{Nb}$ in a combined fashion as $\mathrm{c} / \mathrm{pN} 3$.

\section{Results}

\section{Patients and treatment}

70 patients who underwent surgery with curative intent for supraglottic laryngeal cancer between 2004 and 2019 met the inclusion criteria. $73 \%(n=51)$ of patients were male $27 \%(n=19)$ were female. Age distribution was as follows: $\leq 49$ years: $3 \%(n=2)$; $50-59$ years: $21 \%(n=15)$, 60-69 years: $49 \%(n=34), 70-79$ years: $23 \%(n=16)$ and $\geq 80$ years: $4 \%(n=3) .50 \%(n=35)$ of patients presented with clinical stage $\mathrm{T} 1$ and $\mathrm{T} 2$ primary tumors and $50 \%(n=35)$ of patients presented with stage T3 and T4 primary tumors at the time of diagnosis. Clinically assessed nodal status was $\mathrm{cN} 0$ for $43 \%(n=30)$ of patients, $\mathrm{cN} 1$ for $6 \%(n=4), \mathrm{cN} 2$ for $46 \%(n=32)$ of patients and $\mathrm{cN} 3$ for $6 \%(n=4)$ of patients. According to AJCC (American Joint Committee on Cancer) criteria, $4 \%(n=3)$ of patients were allocated to stage I, $17 \%(n=12)$ to stage II, $21 \%(n=15)$ to stage III and 57\% $(n=40)$ to stage IV. $47 \%(n=33)$ of patients underwent total laryngectomy, while $53 \%(n=37)$ of patients underwent transoral tumor resection (21 patients with TORS, 16 patients with TLM). On post-operative pathology, 55\% $(n=17 / 31)$ of patients with early-stage primary cancers (i.e., pT1/2), had positive lymph nodes after surgical staging, compared to $67 \%(n=26 / 39)$ of patients with advanced stage primary cancers, i.e. pT3/4 cancers. Patient and disease characteristics are provided in Table 1. All patients underwent bilateral neck dissection. One patient had resectable distant metastatic disease and received a lobectomy for lung metastasis. The postoperative tumor board recommended adjuvant therapy in $71 \%(n=50)$ of cases. 
Table 1 Clinical patient characteristics

\begin{tabular}{|c|c|}
\hline \multicolumn{2}{|c|}{$\begin{array}{l}\text { Patient and disease charac- } \\
\text { teristics }\end{array}$} \\
\hline \multicolumn{2}{|l|}{ Gender } \\
\hline Male & $73 \%$ \\
\hline Female & $27 \%$ \\
\hline Age (avg. in years) & 65 \\
\hline \multicolumn{2}{|l|}{ Clinical T stage } \\
\hline cT1 & $10 \%$ \\
\hline cT2 & $40 \%$ \\
\hline cT3 & $34 \%$ \\
\hline cT4 & $16 \%$ \\
\hline \multicolumn{2}{|l|}{ Clinical N stage } \\
\hline cNO & $43 \%$ \\
\hline cN1 & $6 \%$ \\
\hline $\mathrm{cN} 2$ & $46 \%$ \\
\hline $\mathrm{cN} 3$ & $6 \%$ \\
\hline \multicolumn{2}{|l|}{ AJCC stage } \\
\hline I & $4 \%$ \\
\hline II & $17 \%$ \\
\hline III & $21 \%$ \\
\hline IVa & $49 \%$ \\
\hline $\mathrm{IVb}$ & $7 \%$ \\
\hline IVc & $1 \%$ \\
\hline \multicolumn{2}{|l|}{ Surgical treatment } \\
\hline Total laryngectomy & $47 \%$ \\
\hline TORS & $30 \%$ \\
\hline TLM & $23 \%$ \\
\hline Adjuvant therapy & $71 \%$ \\
\hline
\end{tabular}

AJCC American Joint Committee on Cancer, TORS transoral robotic surgery, TLM transoral laser microsurgery

\section{Survival analysis}

A survival analysis was performed (Fig. 1), showing $80 \%$, $58 \%$, and $30 \%$ of patients to be alive after 24,60 , and 135 months of follow-up, respectively.

\section{Clinical vs. pathological nodal staging}

Clinical and pathological nodal status differed in $24 \%$ ( $n=17 / 70)$ of all cases: Upstaging, i.e., $\mathrm{pN}>\mathrm{cN}$ occurred in $16 \%$ of cases $(n=11 / 70)$ and was more common than downstaging, i.e., $\mathrm{pN}<\mathrm{cN}$ which occurred in $9 \%(n=6 / 70)$ of cases (Table 2). Figure 2 visualizes the re-staging occurring between clinical and pathological nodal status. One can appreciate that in our patient collective, the occurrence of single metastases (smaller or larger than $3 \mathrm{~cm}$ ) are less common than multiple metastases in the individual patient. Of note, pathological nodal stage $\mathrm{N} 2 \mathrm{~b}$, i.e., multiple ipsilateral metastatic lymph nodes seems to be most difficult to assess

Overall survival

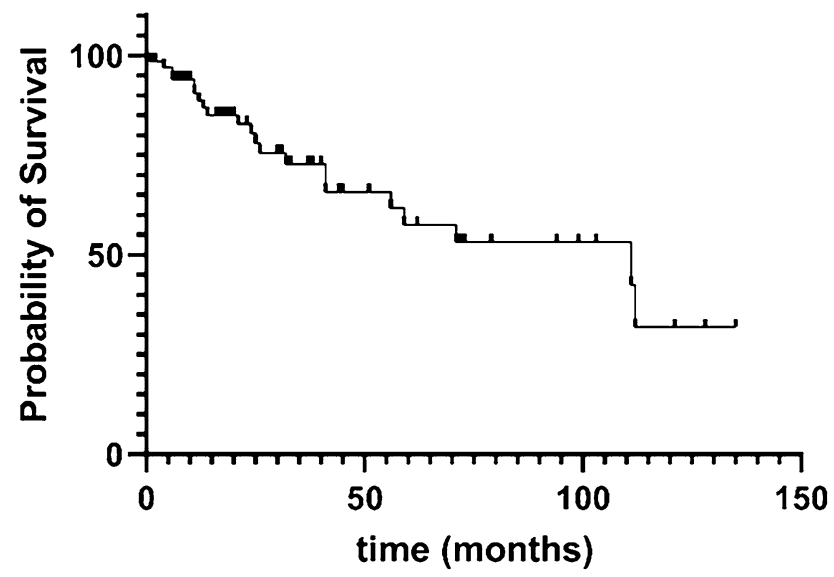

Fig. 1 Kaplan-Meier curve showing overall survival data for 67 patients. At the 24-month cut-off, $80 \%$ of patients were alive, at the 60 -month cut-off, $58 \%$ of patients were alive, at 135 months, $32 \%$ of patients were alive

clinically, as three patients were wrongly classified as $\mathrm{cN} 2 \mathrm{~b}$ while seven patients with $\mathrm{pN} 2 \mathrm{~b}$ were previously classified differently on clinical staging, resulting in a consistency rate of $56 \%$ when comparing clinical to pathological staging.

Changes from cN0 status to $\mathrm{pN}+-$-status are clinically the most relevant and were, therefore, analyzed in detail. $14 \%$ of all patients clinically staged N0 $(n=5 / 30)$ showed metastatic nodes on final pathology and details for these are summarized in Table 2. Remarkably, all metastatic nodes detected in this patient collective were less than $1.6 \mathrm{~cm}$ in diameter. In one case, upstaging from $\mathrm{N} 0$ to $\mathrm{N} 3$ was due to pathological extracapsular spread in one node.

\section{Therapeutic implications of neck staging differences}

Therapeutic implications for all patients where final pathology findings differed from the initial clinical neck staging results (24\% of all patients, $n=17 / 70$ ) are listed in Table 3. All patients in our analysis were treated with primary surgery including bilateral neck dissection and in $76 \%$ $(n=13 / 17)$ of patients where $\mathrm{pN}$ differed from $\mathrm{cN}$, adjuvant treatment was affected the altered neck staging results. We also performed a virtual retrospective tumor board to assess whether the therapeutic regime would have been altered if patients had been treated with primary a primary non-surgical approach and the true nodal status had been known: here, treatment alterations would have occurred in $82 \%(n=14 / 17)$ of patients. Notably, $11 \%(n=8 / 70)$ of all patients would have received more intensive treatment according to current guidelines based on their $\mathrm{pN}$ status compared to their $\mathrm{cN}$ status. 


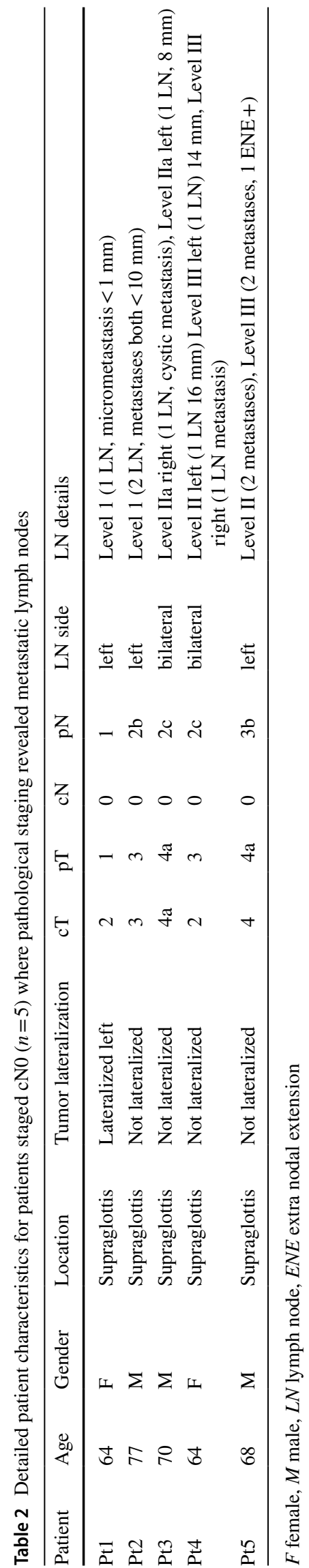

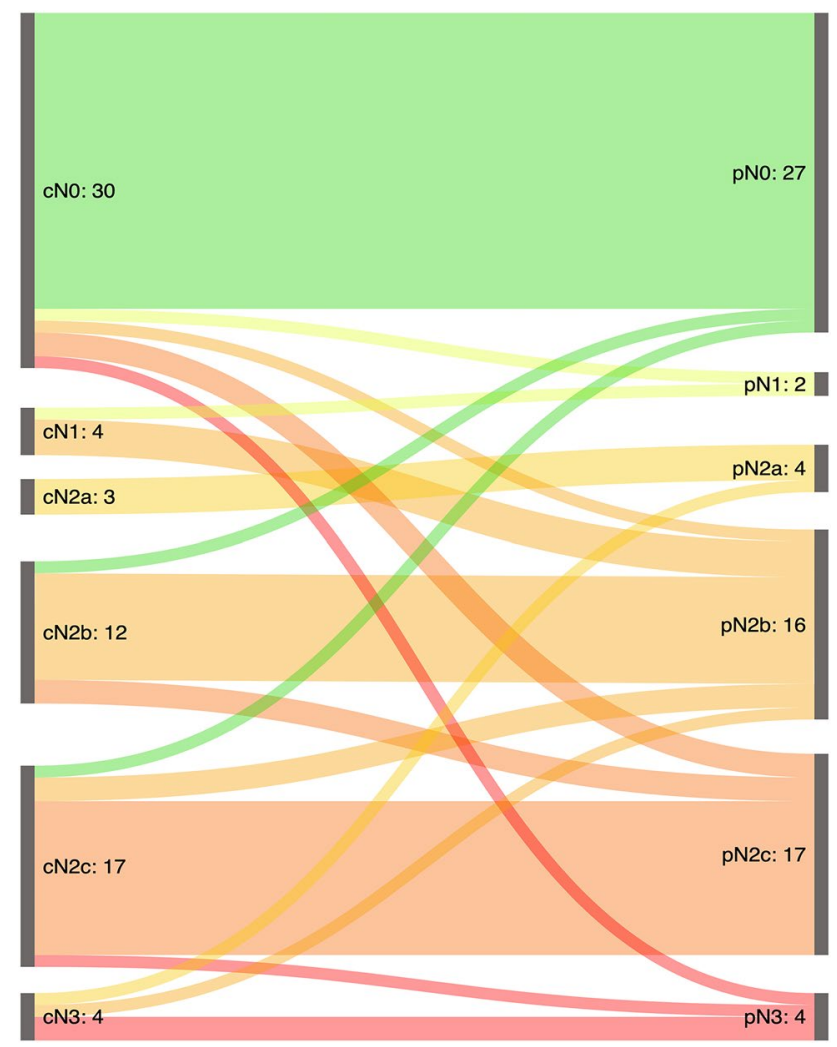

Fig. 2 Sankey diagram graphically depicting the changes from clinical to pathological staging for all patients. Overall, cN stage differed fom $\mathrm{pN}$ stage in $24 \%(n=17 / 70)$ of cases. Upstaging occurred in $16 \%$ of cases $(n=11 / 70)$ and was more common than downstaging, which occurred in $9 \%(n=6 / 70)$ of cases

\section{Discussion}

The findings of our retrospective study confirm the pronounced tendency of supraglottic laryngeal cancer to develop regional metastases at all stages. In our patient collective, patients with early stage primary tumors, i.e, stages $\mathrm{T} 1$ and $\mathrm{T} 2$, developed lymph node metastases in 55\% of cases at the time of surgery, compared to $67 \%$ of patients with advanced local tumors, i.e., T stages 3 and 4 . This is in line with findings by others, who showed similarly high regional metastasis rates [14]. While neck dissection is indisputably part of any primary surgical treatment approach for supraglottic laryngeal cancer and part of current treatment guidelines, unilateral neck dissection for early stage primary tumors with a clinical N0 neck has been advocated [15], since occult nodal metastases are most likely to occur ipsilaterally in patients with $\mathrm{T} 1$ and $\mathrm{T} 2$ primary tumors. In our study, $17 \%(n=5 / 30)$ of patients across all stages who were clinically staged NO had positive lymph nodes on final pathological assessment, i.e., occult nodal metastases. These numbers are comparable, or slightly lower than those reported by other authors, who found occult metastases in 
Table 3 Summary of therapeutic relevance of $\mathrm{c} / \mathrm{pN}$ divergence and resulting changes of adjuvant and primary radiation therapy

\begin{tabular}{llllll}
\hline $\mathrm{cN}$ & $\rightarrow \mathrm{pN}$ & $\begin{array}{l}\text { No. of } \\
\text { patients }\end{array}$ & $\mathrm{Up} /$ downstaging & Effect of $\mathrm{cN} \rightarrow \mathrm{pN}$ change on adjuvant treatment & $\begin{array}{l}\text { Hypothetical effect of } \mathrm{cN} \rightarrow \mathrm{pN} \text { change on } \\
\text { primary radiotherapy treatment }\end{array}$ \\
\hline $\mathrm{cN} 0$ & $\mathrm{pN} 1$ & 1 & $\mathrm{Up}$ & Higher radiation dose ipsilateral side & Higher radiation dose \\
& $\mathrm{pN} 2 \mathrm{~b}$ & 1 & $\mathrm{Up}$ & Higher radiation dose ipsilateral side & Higher radiation dose \\
& $\mathrm{pN} 2 \mathrm{c}$ & 2 & $\mathrm{Up}$ & Higher radiation dose & Higher radiation dose \\
& $\mathrm{pN} 3 \mathrm{~b}$ & 1 & $\mathrm{Up}$ & Higher radiation dose, additional Chemotherapy & Higher radiation dose, additional Chemotherapy \\
$\mathrm{cN} 1$ & $\mathrm{pN} 2 \mathrm{a}$ & 3 & $\mathrm{Up}$ & None & None \\
$\mathrm{cN} 2 \mathrm{~b}$ & $\mathrm{pN} 0$ & 1 & Down & Lower radiation dose ipsilateral side & Lower radiation dose \\
& $\mathrm{pN} 2 \mathrm{c}$ & 2 & $\mathrm{Up}$ & Higher radiation dose contralateral side & Higher radiation dose contralateral side \\
$\mathrm{cN} 2 \mathrm{c}$ & $\mathrm{pN} 0$ & 1 & Down & Lower radiation dose & Lower radiation dose \\
& $\mathrm{pN} 2 \mathrm{~b}$ & 2 & Down & Lower radiation dose & Lower radiation dose contralateral side \\
& $\mathrm{pN} 3 \mathrm{~b}$ & 1 & $\mathrm{Up}$ & Higher radiation dose & Higher radiation dose, additional Chemotherapy \\
$\mathrm{cN} 3$ & $\mathrm{pN} 2 \mathrm{a}$ & 1 & Down & Lower radiation dose & Lower radiation dose \\
& $\mathrm{pN} 2 \mathrm{~b}$ & 1 & Down & None & Lower radiation dose \\
\hline
\end{tabular}

Hypothetical effects in case of a primary radiotherapy treatment approach were assessed via a retrospective virtual tumor board

up to $30 \%$ of patients with clinical N0 status at the time of surgery [16-18]. Our data suggest a positive correlation between the rate of occult nodal metastasis and the size of the primary tumor: Four of the five patients with occult nodal metastasis had advanced stage local disease. The one patient with an early-stage primary tumor had an occult nodal micro-metastasis. Notably, all occult metastases were located ipsilaterally. The single case of occult nodal disease within the group of patients with smaller primary tumors is most likely attributable to the sample size of our patient collective; Zhang et al. who focused on a larger cohort of cN0 supraglottic tumors revealed occult nodal metastasis rates of $13.0 \%$ and $20.5 \%$ for pT 1 and pT2 tumors, respectively [18]. Here too, though, contralateral metastases and contralateral regional recurrence were rare in patients with clearly lateralized, early-stage primary tumors. Importantly, this does not apply to patients where the primary tumor is located close to, or even at the anatomical midline, as has been pointed out before [19]. However, if these anatomical and oncologic criteria are met, i.e., a clearly lateralized early stage primary tumor and clinical N0 status, unilateral neck dissection seems to be justifiable. In all other cases, i.e., primary tumors close to the midline, or advanced stage primary tumors, or clinically positive nodal status, bilateral neck dissection should be performed.

Compared to a non-surgical treatment approach, primary surgery for supraglottic laryngeal cancer has the inherent advantage of providing definitive pathological staging of the neck and the aim of this study was to assess the differences between $\mathrm{cN}$ and $\mathrm{pN}$ stage and analyze the impact of surgical staging of the neck on the therapeutic regimen. In our patient collective, pre-therapeutic clinical $\mathrm{N}$-status and post-surgical N-status as assessed by pathology differed in $24 \%$ of cases. These data are in line with analyses by others for supraglottic laryngeal carcinomas [16]; similar discrepancy rates have also been reported for oropharyngeal cancer patients who underwent primary surgery [20]. Importantly, in $16 \%$ of all patients, $\mathrm{pN}$ stage exceeded $\mathrm{cN}$ stage, i.e., surgery revealed additional neck metastases which elevated the patients' disease stage, according to AJCC guidelines. Notably, staging discrepancies affected treatment: for the majority of upstaged patients, the dose of adjuvant radiation to the neck was higher than it would have been according to clinical N-stage. Similarly, in patients where $\mathrm{pN}$ stage was lower than $\mathrm{cN}$ stage, i.e., $9 \%$ of all patients, the adjuvant therapeutic regimen was also affected, and treatment intensity was reduced based on $\mathrm{pN}$ stage. Of course, for our patients, these deliberations are purely hypothetical, since all patients underwent a primary surgical approach and neck dissection was routinely performed, guaranteeing a definitive pathological neck staging result. However, these findings do serve as an affirmation of the value of surgical staging of the neck, particularly in an era, where more organ-preserving surgical approaches are available for supraglottic laryngeal cancer patients as an alternative to primary radiation. The advancements in transoral surgery, particularly promoted by the recent developments in TORS for supraglottic laryngeal cancer, have provided a surgical, yet organ-preserving primary treatment approach for selected patients who may have otherwise be treated with primary radiation therapy. In our patient cohort, $53 \%$ of patients underwent transoral surgery, the majority via TORS with the Medrobotics ${ }^{\circledR}$ FLEX system, and $21 \%$ of those patients had stage T3 primary tumors, which could be successfully resected via the transoral approach, emphasizing the comprehensiveness of the technology, as has been previously shown [21-23]. For these patients in particular, transoral surgery combined with bilateral neck dissection provided an effective primary treatment 
while allowing for highly accurate adjuvant therapy based on the results of pathologic staging of the neck.

\section{Conclusion}

Our data confirm the notion that supraglottic laryngeal cancers show a pronounced tendency to spread to regional lymph nodes of the neck at every disease stage. Therefore, in case of a primary surgical treatment approach, bilateral neck dissection is warranted with the exception of early stage, clearly lateralized tumors staged cN0; here, ipsilateral neck dissection may be considered. Our analysis revealed a considerable discrepancy between clinical and pathological neck staging results which affected the adjuvant treatment regimen in our patients. Notably, as determined via a retrospective tumor board, if a primary non-surgical treatment approach had been chosen for our patients, nearly $20 \%$ of patients would have received an under/overtreatment of the neck because $\mathrm{cN}$-staging was inaccurate. These findings should be taken into consideration when establishing the primary treatment approach for patients with supraglottic laryngeal cancer, particularly for patients where both surgical and non-surgical treatment approaches are available.

Author contributions Conceptualization: CHLK and TH. Data acquisition and analysis: CHLK; EZ, TG, MG, JML, ED, and TH. Manuscript writing: CHLK and TH. Writing-review and editing: all authors.

Funding Open Access funding enabled and organized by Projekt DEAL. C.H.L.K. was supported by the Essen University IFORES research program and the Essen University UMEA junior clinician scientist program.

\section{Declarations}

Conflict of interest S.M. and S.L. have received speaker fees and travel reimbursements by Medrobotics Corp.

Ethics approval This study was performed in line with the principles of the Declaration of Helsinki. This study was approved by the ethics committee of the University of Essen, Germany (internal reference code: 20-9441-BO).

Open Access This article is licensed under a Creative Commons Attribution 4.0 International License, which permits use, sharing, adaptation, distribution and reproduction in any medium or format, as long as you give appropriate credit to the original author(s) and the source, provide a link to the Creative Commons licence, and indicate if changes were made. The images or other third party material in this article are included in the article's Creative Commons licence, unless indicated otherwise in a credit line to the material. If material is not included in the article's Creative Commons licence and your intended use is not permitted by statutory regulation or exceeds the permitted use, you will need to obtain permission directly from the copyright holder. To view a copy of this licence, visit http://creativecommons.org/licenses/by/4.0/.

\section{References}

1. Liu Y-H, Xu S-C, Tu L-L et al (2006) A rich lymphatic network exists in the inferior surface of the vocal cord. Surg Radiol Anat 28:125-128. https://doi.org/10.1007/s00276-006-0075-2

2. Werner JA, Schünke M, Rudert H, Tillmann B (1990) Description and clinical importance of the lymphatics of the vocal fold. Otolaryngol Head Neck Surg 102:13-19. https://doi.org/10.1177/ 019459989010200103

3. Leitlinienprogramm Onkologie (Deutsche Krebsgesellschaft, Deutsche Krebshilfe, AWMF): Diagnostik, Therapie und Nachsorge des Larynxkarzinoms, Langversion 1.1, 2019, AWMFRegisternummer: 017/076OL, http://www.leitlinienprogr amm-onkologie.de/leitlinien/larynxkarzinom/ (abgerufen am: 19.04.2020)

4. Jones TM, De M, Foran B et al (2016) Laryngeal cancer: United Kingdom National Multidisciplinary guidelines. J Laryngol Otol 130:S75-S82. https://doi.org/10.1017/S00222151160004 87

5. Paleri V, Urbano TG, Mehanna H et al (2016) Management of neck metastases in head and neck cancer: United Kingdom National Multidisciplinary Guidelines. J Laryngol Otol 130:S161S169. https://doi.org/10.1017/S002221511600058X

6. Weiss MH, Harrison LB, Isaacs RS (1994) Use of decision analysis in planning a management strategy for the stage N0 neck. Arch Otolaryngol Head Neck Surg 120:699-702. https://doi.org/ 10.1001/archotol.1994.01880310005001

7. Robbins KT, Medina J, Ferlito A (2019) Adding clarity to the historical evolution of the selective neck dissection. Head Neck 41:1542-1544. https://doi.org/10.1002/hed.25704

8. de Bree R, Takes RP, Shah JP et al (2019) Elective neck dissection in oral squamous cell carcinoma: past, present and future. Oral Oncol 90:87-93. https://doi.org/10.1016/j.oraloncology.2019.01. 016

9. Coskun HH, Medina JE, Robbins KT et al (2015) Current philosophy in the surgical management of neck metastases for head and neck squamous cell carcinoma: Neck Treatment in HNSCC. Head Neck 37:915-926. https://doi.org/10.1002/hed.23689

10. Ferlito A, Robbins KT, Shah JP et al (2011) Proposal for a rational classification of neck dissections. Head Neck 33:445-450. https:// doi.org/10.1002/hed.21614

11. Langendijk JA, Ferlito A, Takes RP et al (2010) Postoperative strategies after primary surgery for squamous cell carcinoma of the head and neck. Oral Oncol 46:577-585. https://doi.org/10. 1016/j.oraloncology.2010.03.023

12. Lavaf A, Genden EM, Cesaretti JA et al (2008) Adjuvant radiotherapy improves overall survival for patients with lymph node-positive head and neck squamous cell carcinoma. Cancer 112:535-543. https://doi.org/10.1002/cncr.23206

13. Bernier J, Cooper JS, Pajak TF et al (2005) Defining risk levels in locally advanced head and neck cancers: a comparative analysis of concurrent postoperative radiation plus chemotherapy trials of the EORTC (\#22931) and RTOG (\# 9501). Head Neck 27:843-850. https://doi.org/10.1002/hed.20279

14. Chiu RJ, Myers EN, Johnson JT (2004) Efficacy of routine bilateral neck dissection in the management of supraglottic cancer. Otolaryngol Head Neck Surg 131:485-488. https://doi.org/10. 1016/j.otohns.2004.04.024

15. Rodrigo JP, Cabanillas R, Franco V, Suárez C (2006) Efficacy of routine bilateral neck dissection in the management of the N0 neck in T1-T2 unilateral supraglottic cancer. Head Neck 28:534-539. https://doi.org/10.1002/hed.20359

16. Esposito EDAVE, Motta S, Cassiano B, Motta G (2001) Occult lymph node metastases in supraglottic cancers of the larynx. 
Otolaryngol Head Neck Surg 124:253-257. https://doi.org/10. 1067/mhn.2001.113146

17. Ma H, Lian M, Feng L et al (2014) Factors contributing to lymph node occult metastasis in supraglottic laryngeal carcinoma cT2T4 N0M0 and metastasis predictive equation. Chin J Cancer Res 26:685-691. https://doi.org/10.3978/j.issn.1000-9604.2014.12.06

18. Zhang Y, Xu S, Liu W et al (2020) Rational choice of neck dissection in clinically N0 patients with supraglottic cancer. Head Neck 42:365-373. https://doi.org/10.1002/hed.26014

19. Ferlito A, Silver CE, Rinaldo A (2008) Selective neck dissection (IIA, III): a rational replacement for complete functional neck dissection in patients with N0 supraglottic and glottic squamous carcinoma. Laryngoscope 118:676-679. https://doi.org/10.1097/ MLG.0b013e31815f6f25

20. Walvekar RR, Li RJ, Gooding WE et al (2008) Role of surgery in limited (T1-2, N0-1) cancers of the oropharynx. Laryngoscope 118:2129-2134. https://doi.org/10.1097/MLG.0b013e3181 857950

21. Hussain T, Lang S, Haßkamp P et al (2020) The Flex robotic system compared to transoral laser microsurgery for the resection of supraglottic carcinomas: first results and preliminary oncologic outcomes. Eur Arch Otorhinolaryngol 277:917-924. https://doi. org/10.1007/s00405-019-05767-0

22. Razafindranaly V, Lallemant B, Aubry K et al (2016) Clinical outcomes with transoral robotic surgery for supraglottic squamous cell carcinoma: experience of a French evaluation cooperative subgroup of GETTEC: Clinical outcomes with transoral robotic surgery for supraglottic SCC. Head Neck 38:E1097-E1101. https://doi.org/10.1002/hed.24163

23. Canis M, Martin A, Ihler F et al (2013) Results of transoral laser microsurgery for supraglottic carcinoma in 277 patients. Eur Arch Otorhinolaryngol 270:2315-2326. https://doi.org/10.1007/ s00405-012-2327-6

Publisher's Note Springer Nature remains neutral with regard to jurisdictional claims in published maps and institutional affiliations. 